\title{
INCREMENTO DE LA EXPRESIÓN DE TLR4 Y EFECTO ANTIOXIDANTE DEL ÁCIDO ACETILSALICÍLICO EN CONEJOS CON DIETA ALTA EN GRASAS
}

INCREASE IN THE EXPRESSION OF TLR4 AND ANTIOXIDANT EFFECT OF ACETYLSALICYL ACID IN RABBITS WITH A HIGH FAT DIET

Ortíz-Reyes, Ana Elenka1. Calderón-Torres, C. Marissa ${ }^{*}$

Unidad de Biomedicina, Facultad de Estudios Superiores Iztacala, Universidad Nacional Autónoma de México. Tlalnepantla, Estado de México, México

Citation: Ortiz-Reyes, AE., Calderón-Torres, MC. (2017) Incremento de la expresión de TLR4 y efecto antioxidante del ácido acetilsalicílico en conejos con dieta alta en grasas, Revista de Salud Pública y Nutrición, 16(2), 1-10. Editor: Esteban G. Ramos Peña, Dr. CS., Universidad Autónoma de Nuevo león, Facultad de Salud Pública, Monterrey Nuevo León, México.

Copyright: (C2017 Ortiz-Reyes, AE et al. This is an open-access article distributed under the terms of Creative Commons Attribution License [CC BYND 4.0], which permits unrestricted use, distribution, and reproduction in any medium, provided the original author and source are credited.

Competing interests: The authors have declared that no competing interests exist.

DOI: https://doi.org/10.29105/respyn16.2-1

*Email: mcalderon@campus.iztacala.unam.mx 


\title{
INCREMENTO DE LA EXPRESIÓN DE TLR4 Y EFECTO ANTIOXIDANTE DEL ÁCIDO ACETILSALICÍLICO EN CONEJOS CON DIETA ALTA EN GRASAS
}

\author{
Ortíz-Reyes, Ana Elenka'. Calderón-Torres, C. Marissa 1*
}

Unidad de Biomedicina, Facultad de Estudios Superiores Iztacala, Universidad Nacional Autónoma de México. Tlalnepantla, Estado de México, México

\begin{abstract}
RESUMEN
Introducción: La obesidad y el desarrollo de enfermedades hepáticas que se caracterizan por el aumento y acumulación de lípidos en tejidos y sangre, inflamación y estrés oxidante, son actualmente una epidemia mundial, y en la población mexicana es cada vez mayor el número de jóvenes afectados. Este aumento ha conducido a la investigación médica hacia la detección temprana del síndrome metabólico, que se emplea como indicador de síntomas que pueden ser de riesgo para la salud y conducir a enfermedades hepáticas. Objetivo: Los objetivos del presente trabajo fueron: evaluar en un modelo de dislipidemia en conejos jóvenes alimentados con una dieta alta en grasa (ácido palmítico al 20\%), la producción de especies reactivas del oxígeno y cambios en la expresión de genes $T L R 4$, COX2y de $L L-1 \beta$ como marcadores de inflamación y de estrés oxidante, así como evaluar el efecto del ácido acetilsalicílico en la producción de radicales libres y en la expresión de estos genes. Resultados: En los conejos alimentados con exceso de grasa aumentaron los niveles de triglicéridos ( $p<0.05$ ), la expresión de TLR4 y las especies reactivas del oxígeno, aunque éstas últimas no de forma significativa. La administración de ácido acetilsalicílico en dosis antiinflamatorias disminuyó la producción de especies reactivas del oxígeno y la expresión de TLR4. Discusión: La ingesta elevada de grasa en conejos jóvenes por un período corto de tiempo conduce a la dislipidemia y a la sobreexpresión de TLR4, gen clave de la respuesta inflamatoria y vinculada al aumento de las especies reactivas del oxígeno. Los resultados indican que el ácido acetilsalicílico tiene efecto antioxidante.
\end{abstract}

Palabras Clave: Obesidad, dislipidemia, inflamación, estrés oxidante, hígado graso no alcohólico (HGNA).

\section{ABSTRACT}

Introduction: The obesity and liver diseases progression are characterized by the increase and accumulation of lipids in tissues and blood, inflammation and oxidative stress. These diseases are now a worldwide epidemic, and the number of young people affected is increasing in the Mexican population. This increase has led to medical research towards the early detection of the metabolic syndrome, which is used as an indicator of symptoms that may be at risk for health and lead to liver disease. Objective: The objectives of the present study were to evaluate in a model of dyslipidemia in young rabbits fed three months with a diet high in fat (20\% palmitic acid), the production of reactive oxygen species and changes in $T L R 4, \operatorname{COX} 2$ and $L L-1 \beta$ gene expression, as markers of inflammation and oxidative stress; also to evaluate the effect of acetylsalicylic acid on the production of free radicals and on the expression of these genes. Results: In rabbits fed with excess of fat, significantly increased the levels of triglycerides $(p<0.05)$, TLR4 expression, and reactive oxygen species, although in the latter, not significantly. The administration of acetylsalicylic acid in anti-inflammatory doses decreased the production of reactive oxygen species and the expression of TLR4. Discussion: The high fat intake in young rabbits lead to dyslipidemia and overexpression of TLR4, a key gene in the inflammatory response and linked to the increase of reactive oxygen species. The results indicate that acetylsalicylic acid has an antioxidant effect

Key words: Obesity, dyslipidemia, inflammation, oxidative stress, non-alcoholic fatty liver (HGNA) 


\section{Introducción}

La obesidad y las enfermedades hepáticas como el hígado graso no alcohólico (HGNA) o la esteatosis no alcohólica, se caracterizan por la acumulación excesiva de lípidos en las células hepáticas (esteatosis), así como una condición de inflamación y de estrés oxidante (Petrosillo et al., 2007). En diferentes modelos biológicos de la enfermedad se ha reportado que la inflamación implica cambios en la expresión genética de las citocinas proinflamatorias TNF- $\alpha$, IL-6, IL-1 $\alpha$, IL-1 $\beta$ y IL-18 (Stojsavljević et al., 2014). Aunado a esto, los productos de la cascada de señalización de inflamación como la ciclo-oxígenasa 2 (COX2) y los receptores de membrana plasmática tipo Toll (TLR), especialmente TLR3 y TLR4 pueden amplificar dicha condición (Liu et al., 2014).

Las citosinas que favorecen la inflamación, representan un grupo diverso, son secretadas por varios tipos de células y tienen diferentes funciones y efectos biológicos. En el estudio de la patogénesis del HGNA destaca la investigación de la citosinas, TNF$\alpha$, IL-6 y las de la familia de IL-1 (Tilg, 2010). TNF$\alpha$ es una molécula secretada por macrófagos infiltrados en el tejido adiposo y en los hepatocitos y es concurrente en la inflamación crónica (Stojsavljević et al., 2014). La citosina IL-6 es producida por adipocitos, hepatocitos, células del sistema inmunológico y endoteliales. En la obesidad el aumento de IL-6 favorece la inflamación de bajo grado, sin embargo su estudio es controversial ya que además de favorecer la inflamación se ha demostrado que tiene efectos protectores del hígado; mientras que la citosina IL-1B, miembro de la familia de las citosinas IL-1, es producida por macrófagos en respuesta al aumento de lipopolisacáridos y ácidos grasos libres (Stojsavljević et al., 2014), y su sobreexpresión ha sido relacionada con el aumento de COX2 (Akarasereenont et al., 1995), esta enzima cataliza la conversión de ácido araquidónico a prostanoides y tromboxanos, moléculas que favorecen la inflamación (Yu et al., 2006).

La expresión de los receptores de membrana es indispensable en la respuesta inmunológica a organismos patógenos, pues reconocen lipopolisacáridos de las bacterias y activan vías de señalización que inducen a la transcripción de citocinas pro-inflamatorias, de la enzima de síntesis de óxido nítrico (iNOS) y de moléculas de adhesión como ICAM y VCAM-1 (Goud y Frienman, 2010). Sin embargo, la sobre-expresión del gen TLR4 en la inflamación de enfermedades hepáticas nada tiene que ver con una respuesta a un organismo patógeno; en su lugar se ha sugerido como una respuesta alterna del sistema inmunológico ante el exceso de lípidos. Se ha propuesto que los ácidos grasos libres oxidados pueden ser reconocidos por TLR4, lo que a su vez activa la vía de señalización de citocinas proinflamatorias (Lee et al., 2003; Suganami et al. 2007; Manček-Keber et al., 2015).

La situación de estrés oxidante ante una dieta rica en ácidos grasos se favorece por la sobre-producción de especies reactivas del oxígeno (ERO), por diferentes procesos o reacciones, como la peroxidación de lípidos (Oliveira et al., 2002) o por el aumento de actividad enzimática de la acetil-CoA deshidrogenasa (VLCAD) (Cardoso et al., 2013). Cuando la producción de ERO rebasa la capacidad de los sistemas antioxidantes de la célula, se produce un desequilibrio denominado estrés oxidante. De acuerdo con la teoría celular del envejecimiento, el exceso de radicales libres puede alterar o dañar las funciones de las biomoléculas y su acumulación continua puede modificar primero las funciones celulares y posteriormente la de órganos y tejidos (Kourtis y Tavernarakis, 2011).

Por ello, en las células hepáticas, la continua presencia de niveles elevados de ácidos grasos libres, que pueden conducir a estrés oxidante y a inflamación, es un factor determinante para el progreso hacia la enfermedad del HGNA. Por consiguiente, esta enfermedad se ha considerado como una de las manifestaciones del denominado síndrome metabólico, definido como un conjunto de síntomas independientes y de riesgo para la salud, como la presión arterial elevada, aumento de la concentración de glucosa y de lípidos (dislipidemia) en sangre, especialmente de triglicéridos, niveles bajos de HDL, así como obesidad central, es decir, acumulación de grasa en la cintura (Alberti et al., 2009).

La enfermedad del HGNA sin atención médica oportuna puede conducir a cirrosis o en casos más graves a cáncer hepático. Aunque se han hecho diferentes trabajos concernientes a la búsqueda de marcadores moleculares tempranos de dislipidemia o esteatosis (Pagadala et al., 2009), aún no se ha 
determinado cuál puede ser el mejor marcador que determine su diagnóstico temprano.

Por ello, las investigaciones de HGNA se han centrado tanto en la búsqueda de marcadores moleculares tempranos que puedan definir alguna de las alteraciones antes mencionadas, así como en el uso de compuestos que disminuyan o inhiban el desarrollo de esteatosis, sin efectos colaterales adversos. De los diversos compuestos estudiados, destacan los trabajos con estatinas, como el caso de la atorvastatina que reduce la expresión del gen TLR4 (Fang et al., 2014); la vitamina $\mathrm{C}$, que disminuye los niveles de triglicéridos en sangre (McRae, 2008); y los compuestos antiinflamatorios no esteroideos, por ejemplo el ácido aceitesalicílico (AAS), que inhibe específicamente a la enzima COX2 (Clària et al., 1996), en consecuencia se bloquea la producción de prostanoides, prostanglandinas y tromboxanos (Ricciotti et al., 2011), además de ser reportado como antioxidante (Mahmood et al., 2009).

Considerando lo anterior, el objetivo de este trabajo fue investigar el cambio en la expresión de los genes $T L R 4, C O X 2$ y de $I L-1 \mathrm{f} 3$, como indicadores de estrés oxidante por lípidos, y como posibles marcadores de cuadros inflamatorios en obesidad, así como evaluar si la administración del ácido acetilsalicílico (AAS) en dosis antiinflamatoria tiene un efecto en la producción de especies reactivas del oxígeno y en la expresión genética de TLR4, COX2 y de IL-1f3.

\section{Material y Métodos \\ Obtención de las muestras biológicas}

En este trabajo se utilizaron conejos gazapos de la cepa Nueva Zelanda (Oryctologus cuniculus) de 3 meses de edad, provenientes de la granja de la Facultad de Veterinaria y Zootecnia de la Facultad de Estudios Superiores Cuautitlán, UNAM. Los conejos se mantuvieron en el Bioterio de la Facultad de Estudios Superiores Iztacala, UNAM en condiciones controladas de crecimiento, es decir, a una temperatura de $23 \pm 5^{\circ} \mathrm{C}$, con un ciclo de luz/obscuridad de $12 \mathrm{~h}$ cada uno y humedad relativa del ambiente de 45 a $60 \%$. Los conejos se dividieron en cuatro grupos experimentales de 6 organismos cada uno: A) Grupo Control (CN), con libre acceso a agua y alimento comercial, de composición balanceada de nutrimentos para dietas de crecimiento de conejos jóvenes; B) Grupo Grasa (AP) a cuyo alimento se agregó $20 \%$ de ácido palmítico; C) Grupo Grasa-Ácido acetilsalicílico (AP/AAS) de libre acceso a comida con $20 \%$ ácido palmítico y la administración de una dosis antiinflamatoria de ácido acetilsalicílico $28.5 \mathrm{mg} / \mathrm{kg}$ (Cyrus et al., 2002; PaulClark et al., 2004); y D) Grupo con administración de ácido acetilsalicílico (AAS) con libre acceso a comida y la administración de ácido acetilsalicílico (28.5 $\mathrm{mg} / \mathrm{kg}$ ). Los tratamientos se suministraron diariamente durante 3 meses. Los animales fueron sacrificados con una sobredosis de pentobarbital sódico $(63 \mathrm{mg} / 2.5 \mathrm{~kg}$ de peso) y se realizó la disección, del hígado se obtuvieron muestras que se colocaron en tubos cónicos de polipropileno de $2 \mathrm{ml}$, estos se congelaron con nitrógeno líquido y se almacenaron en un ultracongelador a $-70^{\circ} \mathrm{C}$.

\section{Cuantificación de lípidos}

Durante el sacrificio de los conejos se obtuvieron muestras sanguíneas para determinar las concentraciones plasmáticas de colesterol total, HDL, LDL y triglicéridos. Las muestras se analizaron en el laboratorio de referencia internacional Grupo Diagnóstico Médico PROA S.A. de C.V. (Laboratorio CARPERMOR). A partir de los datos de concentración de lípidos se calculó el promedio de cada grupo experimental y se obtuvo el error típico, los datos se analizaron con la prueba de $t$ de Student. Las diferencias con un valor de $\mathrm{p}<0.05$ se consideraron significativas.

Cuantificación de las especies reactivas de oxígeno (ERO) totales en extractos celulares

La evaluación de ERO se hizo de acuerdo al método modificado de Hempel et al. (1999); el extracto celular se obtuvo de acuerdo con el ensayo modificado de Song et al. (2013). De cada muestra de hígado se tomó $100 \mathrm{mg}$ y para eliminar el exceso de sangre se lavó con buffer de fosfatos (PBS) 1X; el tejido se transfirió a un tubo cónico de propileno de 2 $\mathrm{mL}$ y se le añadió $500 \mu \mathrm{l}$ de PBS $1 \mathrm{X}$. La lisis celular se hizo con micropistilo de teflón y $5 \mu \mathrm{l}$ de fluoruro de fenilmetilsulfonilo (PMSF) $100 \mathrm{mM}$. El macerado se centrifugó a $13000 \mathrm{rpm}$ por $3 \mathrm{~min}$ a $4^{\circ} \mathrm{C}$. La fase acuosa se transfirió a un tubo nuevo y se mantuvo en hielo. Luego una alícuota de $50 \mu 1$ de cada muestra, por triplicado, se colocó en una placa de 96 pozos y se añadió $195 \mu \mathrm{l}$ de PBS $1 \mathrm{X}$ y $5 \mu \mathrm{l}$ de diacetato de 2',7'- diclorofluoresceína (DCFDA) $500 \mu \mathrm{M}$. La placa se colocó en un fluorómetro con lector de 
placas de 96 pozos, la fluorescencia de las muestras se registró a una longitud de onda de excitación de $485 \mathrm{~nm}$ y de emisión de $520 \mathrm{~nm}$, por $60 \mathrm{~min}$. El valor de fluorescencia se reportó como la unidad relativa de fluorescencia por $\mathrm{mg}$ de proteína total del extracto celular. La concentración de proteína se determinó con el método de espectrofotometría de luz UV a $280 \mathrm{~nm}$ y una curva estándar de albumina de suero de bovino (BSA). De los datos de fluorescencia relativa de cada grupo experimental, se calculó el promedio y se obtuvo el error típico, los datos se analizaron con la prueba de $t$ de Student. Las diferencias con un valor de $\mathrm{p}<0.05$ se consideraron significativas.

\section{Extracción de ARN total por Fenol ácido}

El aislamiento del ARN total se hizo de acuerdo al método de Schmitt et al. (1990) previamente modificado (Calderón-Torres et al., 2006). De cada hígado se tomó una muestra de 0.1 gramos, que se lavó y cortó en trozos pequeños; luego se transfirió a un tubo cónico de propileno de $2 \mathrm{~mL}$ estéril y se le añadió $500 \mu \mathrm{l}$ de buffer $\mathrm{AE}$ [Acetato de sodio $50 \mathrm{mM}$ y EDTA $10 \mathrm{mM}$, pH 5.3] y para inhibir la actividad de enzimas que degradan ARN, se añadió $5 \mu \mathrm{l}$ de dietil pirocarbonato (DEPC); el tubo se agitó suavemente, y el tejido macerado se transfirió a otro tubo cónico de propileno de $2 \mathrm{~mL}$ calentado a $65^{\circ} \mathrm{C}$, que contenía $400 \mu 1$ de fenol ácido, $20 \mu 1$ de SDS al $20 \%$ y perlitas de vidrio; el tubo se incubó en baño maría a $65^{\circ} \mathrm{C}$ por 5 min y se agitó en el vórtex por 30 $\mathrm{s}$; este paso se repitió dos veces, y después el tubo se enfrió a $-70^{\circ} \mathrm{C}$ por 5 min y se centrifugó a $13000 \mathrm{rpm}$ por $5 \mathrm{~min}$. La fase acuosa se transfirió a un tubo nuevo y se le agregó $400 \mu \mathrm{l}$ de fenol; se agitó en vórtex y se centrifugó a $13000 \mathrm{rpm}$ por $5 \mathrm{~min}$; nuevamente la fase acuosa se transfirió a otro tubo cónico de propileno de $2 \mathrm{~mL}$ y se añadió $250 \mu \mathrm{l}$ de fenol y $240 \mu \mathrm{l}$ de la mezcla cloroformo-isoamílico, se agitó en vórtex y se centrifugó a 13000 rpm. El

Tabla I. Secuencia de los oligonucleótidos de anclaje de cada gen.

\begin{tabular}{|c|c|c|c|c|}
\hline \multirow[b]{2}{*}{ Gen } & \multirow[b]{2}{*}{$\begin{array}{l}\text { Secuencia de } \\
\text { referencia (NCBI) }\end{array}$} & \multicolumn{2}{|l|}{ Secuencia de oligonucleótidos } & \multirow[b]{2}{*}{$\begin{array}{l}\text { Tamaño del } \\
\text { amplificado }\end{array}$} \\
\hline & & $\begin{array}{l}\text { (F_oligo 5'->3'cadena sentido "Forward' } \\
\text { >3'cadena de sentido complementario }\end{array}$ & $\begin{array}{l}\text { R_oligo 5'- } \\
\text { Reverse") }\end{array}$ & \\
\hline $\begin{array}{l}\text { Gliceraldehido-3- } \\
\text { fosfato } \\
\text { deshidrogenasa }\end{array}$ & NM_001082253.1 & $\begin{array}{l}\text { F- TGACGACATCAAGAAGGTGC } \\
\text { R- GAAGGTGGAGGAGTGGGTC }\end{array}$ & & $121 \mathrm{pb}$ \\
\hline $\begin{array}{l}\text { (GAPDH } \\
\text { Interleucina ) }\end{array}$ & NM_001082201.1 & $\begin{array}{l}\text { F- TCTGCAACACCTGGGATGA } \\
\text { TCAGCTCATACGTGCCAGAC }\end{array}$ & F- & $114 \mathrm{pb}$ \\
\hline $\begin{array}{l}(I L-1 \beta) \\
\text { Ciclooxigenasa }\end{array}$ & NM_001082388.1 & $\begin{array}{l}\text { TTGACCAGTACAAGTGCGAC } \\
\text { AGTGCGTAAGGATGTAGTGC }\end{array}$ & $\begin{array}{l}\text { R- } \\
\text { F- }\end{array}$ & $132 \mathrm{pb}$ \\
\hline $\begin{array}{l}(C O X-2) \\
\text { Receptor tipo }\end{array}$ & NM_001082732.2 & $\begin{array}{l}\text { GTGGTATCTTTTGCTGTAGTT } \\
\text { TTCTCACCCAGTCCTCATCC }\end{array}$ & R- & $142 \mathrm{pb}$ \\
\hline
\end{tabular}

ARN de la fase acuosa se precipitó con acetato de sodio $3 \mathrm{M}$ y etanol absoluto y se incubó a $-70^{\circ} \mathrm{C}$ por 30 min. Posteriormente el tubo se centrifugó por 15 min a $13000 \mathrm{rpm}$; el sobrenadante se descartó y el ARN precipitado se lavó dos veces con etanol al 75\%. Una vez evaporado el etanol, el ARN se resuspendió cuidadosamente en agua con DEPC y se almacenó a $-70^{\circ} \mathrm{C}$. La pureza e integridad del ARN se verificó mediante electroforesis en gel de agarosa (1\%) y formaldehído. El ADN remanente se eliminó con un sistema comercial de ADNasa (Ambion RNA, life Technologies). La concentración de ARN se determinó mediante espectrofotometría con luz UV a $260 \mathrm{~nm}$.

\section{Diseño de oligonucleótidos}

Para amplificar el ARN mensajero de los genes $G A P D H$, Interleucina $I L-1 \beta$, Ciclooxigenasa 2 $(C O X 2)$ y el receptor tipo Toll 4 (TLR4), primero se obtuvo la secuencia del ARN mensajero de cada gen en la base de datos del National Center for Biotechnology Information (NCBI) y a partir de cada secuencia se diseñó un par de oligonucleótidos con los programas Primer del NCBI (http://biotools.nubic.northwestern.edu/OligoCalc) y Primer3 v.0.4.0 (Untergasser A, 2012). La secuencia de oligonucleótidos específicos para cada gen se muestra en la tabla I. La especificidad teórica de cada par de oligonucleótidos se calculó con los programas disponibles en internet OligoCalc (Kibbe, 20017) y OligoAnalyzer (Integrated DNA Technologies, 2017). Los oligonucleótidos se sintetizaron en la Unidad de Biología Molecular del Instituto de Fisiología Celular, UNAM. 
Obesidad, dislipidemia, inflamación, estrés oxidante, hígado graso no alcohólico (HGNA).

\section{RT-PCR-tiempo real}

La expresión de los genes TLR4, COX2e IL-1f3 se cuantificó con el sistema comercial de RT-PCR SYBR Green Power SYBR Green RNA-to- $\mathrm{C}^{\mathrm{TM}}$ 1Step (Applied Biosystems); en este sistema la primer reacción de la transcripción inversa del ARN mensajero (RT) que produce copias de ADN complementario, está acoplada a la reacción en cadena de la polimerasa (PCR) con esta segunda reacción se amplifica y cuantifica el número de copias del ADN complementario.

Para determinar la cantidad óptima necesaria de ARN a usar en cada reacción de RT-PCR y conocer la eficiencia de amplificación de cada par de oligonucleótidos, se hizo una curva de dilución de la concentración del ARN total por muestra de cada grupo experimental y se obtuvo su curva de disociación y estándar. Finalmente, para cada muestra de ARN, se hizo una reacción de RT por triplicado, con una concentración de ARN total de $10 \mathrm{ng} / \mathrm{ul}$ y de cada par oligonucleótidos de $900 \mathrm{nM}$, en un volumen final de reacción de $10 \mu \mathrm{l}$. La reacción de RT-PCR se hizo en el equipo de tiempo real Step One Plus ${ }^{T M}$ (Applied Biosystems).

La temperatura y el tiempo de cada ciclo se mantuvieron de acuerdo con las recomendaciones del proveedor. De cada reacción de RT-PCR se obtuvo su curva disociación y su valor de CT. Para determinar la expresión diferencial de cada gen en las muestras del control $(\mathrm{CN})$ y de los tratamientos (AP, AP/AAS y AAS), se utilizó el método de comparación denominado doble delta $\mathrm{CT}\left(2^{-\triangle \triangle C T}\right)$; con este método, primero se obtuvo la dispersión de los datos del valor CT de los genes inducibles $T L R 4, I L-1 \mathrm{f} 3$ y $C O X 2$, con respecto al valor de CT del gen constitutivo GADPH.

Posteriormente se calculó la diferencia de los valores de CT del control y de los tratamientos para cada gen y el valor de doble delta CT se obtuvo con el logaritmo de la diferencia antes señalada.

Finalmente se calculó el error total del método doble delta CT con la fórmula del coeficiente de variación del boletín de cuantificación relativa de la expresión genética, del proveedor Applied Biosystems (2001).

\section{Resultados \\ Perfil de lípidos en sangre}

Para determinar si los conejos jóvenes alimentados con un exceso de grasa por tres meses tenían dislipidemia, caracterizada por niveles elevados de triglicéridos y de colesterol LDL y la disminución de los valores de colesterol HDL, se obtuvo el perfil de lípidos de los conejos. Al comparar el valor promedio de cada determinación de lípidos en los conejos con dieta normal sin grasa (CN) y de los conejos alimentados con ácido palmítico al 20\% (AP), se observó en estos últimos que la concentración de triglicéridos aumentó significativamente $(\mathrm{p}=0.0465)$; por lo que respecta al valor de LDL, aunque se comprobó que aumentó, este cambio no fue estadísticamente significativo (Figura 1) al igual que la disminución del valor de HDL. En los de conejos alimentados con dieta alta en grasa y administración de ácido acetilsalicílico (AP/AAS), se observó que la concentración de triglicéridos y de LDL disminuyó con respecto al grupo AP y que la de LDL aumentó, pero ninguno de los cambios fue estadísticamente significativo (Figura 1). En los conejos con dieta normal y administración de ácido acetilsalicílico (AAS), el valor de triglicéridos fue similar al del grupo $\mathrm{CN}$ y los valores de HDL y de LDL disminuyeron con respecto al grupo $\mathrm{CN}$, pero estos cambios tampoco fueron estadísticamente significativos.

Figura 1. Perfil de lípidos en sangre de conejos, alimentados por tres meses con dieta normal (CN), ácido palmítico 20\% (AP) y con un suplemento de ácido acetilsalicílico (APIAAS) y con dieta normal + ácido acetilsalicílico (AAS). Las mediciones representan el promedio de los valores obtenidos por cada conejo (conejos control $n=6$ y conejos para cada tratamiento $n=5$ ) con su valor de error típico, la diferencia significativa de los niveles de triglicéridos se indica con un asterisco $(p<0.05)$.

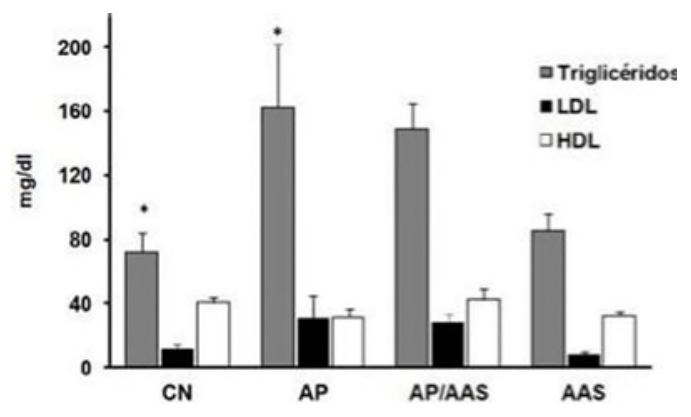


Obesidad, dislipidemia, inflamación, estrés oxidante, hígado graso no alcohólico (HGNA).

\section{Producción de radicales libres totales}

Considerando que los conejos jóvenes alimentados con exceso de grasa presentaron dislipidemia, condición en la que ya se ha reportado estrés oxidante (Furukawa et al., 2004), en las muestras de hígado se midió el nivel de las especies reactivas del oxígeno (ERO). En general los cambios del valor promedio de ERO de cada grupo tratamiento con respecto al grupo control (CN), no fueron estadísticamente significativos, sin embargo se encontró que el valor de ERO de los conejos AP fue el más alto en relación al valor de los conejos CN (Figura 2); mientras que el valor de ERO de los conejos AP/AAS fue similar al del grupo $\mathrm{CN}$, es decir, que en presencia del ácido acetilsalicílico (AAS) disminuyó el nivel de ERO. Por el contrario, en los hígados de los conejos AAS se observó que el nivel de ERO aumentó casi dos veces más que el valor del grupo $\mathrm{CN}$.

Figura 2. Niveles de ERO totales en extractos celulares de hígados de conejos, alimentados por tres meses con dieta normal (CN), ácido graso palmítico $20 \%$ (AP) y con un suplemento de ácido acetilsalicílico (APIAAS) y con dieta normal + ácido acetilsalicílico (AAS). Las mediciones son el promedio de dos lecturas después de $60 \mathrm{~min}$ de reacción con la 2'7' DHFC, ( $n=3$ de conejos CN y APIAAS y $n=2$ de conejos AP y ASS) con su valor de error típico.

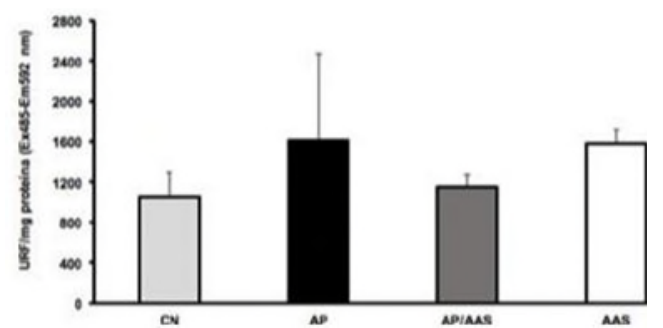

Expresión genética de $C O X 2, I L-1 / 3$ y $T L R 4$

Para evaluar si el exceso de lípidos y la administración de ácido acetilsalisílico en la dieta de los conejos jóvenes tenían un efecto en la expresión de tres genes que codifican para las moléculas marcadoras de inflamación, interleucina $1 \beta(I L-1 / 3)$, enzima ciclo-oxigenasa $2(\mathrm{COX} 2)$ y el receptor de membrana tipo Toll (TLR4), se utilizó la técnica RTPCR cuantitativo. Sorpresivamente se encontró que en las muestras de hígado de los tres grupos de tratamiento, la expresión de $C O X 2$ y de $I L-1 \beta$ fue menor en relación al grupo control $(\mathrm{CN})$. Mientras que la expresión del gen TLR4 en los hígados de los conejos a cuyo alimento se agregó $20 \%$ de ácido palmítico (AP), aumentó hasta seis veces más que en los conejos CN; en los conejos AP/AAS la expresión del gen TLR4 disminuyó en relación con los conejos AP; se consideró que este cambio era debido a la administración de ácido acetilsalisílico (AAS). Por lo que respecta a los conejos del grupo AAS, la expresión del gen TLR4 aumentó hasta cuatro veces más que en los conejos $\mathrm{CN}$.

Figura 3. Niveles relativos del ARN mensajero de tres genes indicadores de inflamación y estrés oxidante en muestras de hígado de conejos, alimentados con ácido palmítico al $20 \%$ (AP), con ácido palmítico + ácido acetilsalicílico (APIAAS), y con dieta normal + ácido acetilsalicílico (AAS). Los niveles de los transcritos se determinaron por RT-PCR en tiempo real. Para cada gen los niveles de expresión de los conejos alimentados con dieta normal (CN) se tomaron como valor de referencia. Los datos se presentan como el valor de $2-\Delta \Delta C T$ y su coeficiente de variación de seis mediciones por cada gen para dos conejos por tratamiento.

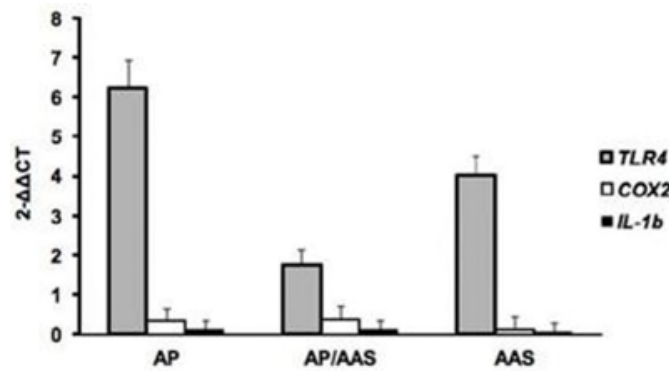

\section{Discusión}

En México, el aumento alarmante de la población de jóvenes con obesidad representa un problema de salud pública que ha generado diferentes estrategias del sector salud para reducir y prevenir este problema, así como investigación enfocada a los componentes del síndrome metabólico en jóvenes y adultos. Lo anterior con el objetivo de evaluar si en etapas tempranas se puede detectar y detener su progresión hacia esteatosis, que a largo plazo podría conducir a la enfermedad del hígado graso no alcohólico (HGNA).

En particular, en los modelos biológicos de estudio de la obesidad, se han buscado marcadores 
tempranos de dislipidemia, siendo los valores arrojados en un análisis de lípidos en sangre, como el aumento de los niveles de triglicéridos y la disminución de HDL los más empleados para indicar que puede haber síndrome metabólico. Tal como se observó en este trabajo, en los conejos jóvenes alimentados con una dieta alta en ácido palmítico (AP), se encontró que en efecto ya tenían dislipidemia, pues los valores de triglicéridos y LDL aumentaron y el valor de HDL disminuyó hasta 3 veces con respecto a las muestras de los conejos con dieta normal $(\mathrm{CN})$.

En los conejos AP + ácido acetilsalicílico (AP/AAS) no se encontró dislipidemia, es probable que en estos conejos la administración de AAS evitó el establecimiento de dislipidemia. De este hallazgo surgió la pregunta ¿cuál es el efecto molecular de AAS que conduce a la reducción de lípidos en sangre? La disminución podría ser debida a la capacidad antioxidante de AAS, que ya ha sido reportada en células del endotelio de arteria pulmonar de bovino (Podhaiski et al., 1997) y en el gusano Caenorhabditis elegans (Ayyderava et al., 2011) ambos expuestos a compuestos oxidantes, así como en la reducción de colesterol en sangre de ratas deficientes en cobre (Fields et al., 2001).

Por ello, en el modelo de dislipidemia del presente trabajo, se decidió evaluar, primero si en los conejos alimentados con el exceso de lípidos (grupo AP), aumentaba la producción de ERO que pudiera conducir al estrés oxidante, y segundo, si la administración de AAS en los conejos AP/AAS disminuía la producción de ERO, tal como ocurre con la mayoría de los antioxidantes. Al respecto, se encontró que en los conejos alimentados con ácido palmítico 20\% (AP), aumentó la producción de ERO en hígado, este resultado es similar al reportado en el hígado de ratas Wistar alimentadas con tocino, papas fritas, chocolates y galletas (Milagro et al., 2006). Por el contrario, se encontró que los niveles de ERO disminuyeron en los conejos AP/AAS. Estos resultados indican, primero, que en la dieta rica en grasa hubo una sobre-producción de ERO, que eventualmente podría resultar en una condición de estrés oxidante, y segundo, indican que la administración de AAS tiene un efecto antioxidante, ya que en los hígados con exceso de grasa disminuyó la producción de ERO.
Al medir la producción de ERO en los conejos AAS se encontró que tenía la misma sobre-producción que en el grupo (AP); es probable que en los conejos de dieta normal con AAS, su efecto sea pro-oxidante, ya que en el hígado el AAS puede ser metabolizado a salicilatos $y$ se ha demostrado en mitocondrias aisladas que los salicilatos pueden desacoplar la cadena de transporte de electrones y la fosforilación oxidativa y en consecuencia al aumento de ERO (Battaglia et al., 2005).

Considerando que la presencia elevada de lípidos en hepatocitos activa una respuesta de transcripción de genes marcadores de inflamación (Stojsavljević et al., 2014), en el presente modelo de conejos jóvenes con dislipidemia, se midió la expresión de los genes $C O X 2$ y de $I L-13$ y TLR4. En el caso de los genes $C O X 2$ y de $I L-13$, se esperaba que su expresión aumentara en el hígado de los conejos AP y que disminuyera tanto en los conejos AP/AAS como en los conejos AAS, pero no se encontró ningún cambio de la expresión de los genes $C O X 2$ y de $I L$ 13 en los hígados de estos grupos. Este resultado es similar a lo reportado por Hsieh et al. (2013), que en ratas Sprague-Dawley alimentadas por tres meses con una dieta alta en grasas $(45 \%)$, no encontraron cambios en la expresión de $C O X 2$ en hígado, solamente en el tejido graso. Es probable que en el modelo de dislipidemia temprana en conejos jóvenes, los cambios en la expresión de los genes $C O X 2$ y de $I L-13$, sólo se observen en macrófagos de sangre periférica y no en hígado, tal como ocurre en macrófagos aislados de sangre de conejos con hiperlipidemia (Chen et al., 2013).

Por lo que respecta a la expresión del gen $T L R 4$, se encontró que aumenta en los hígados de los conejos AP, esto nos conduce a proponer que en conejos jóvenes con dietas altas en grasa, hay una correlación entre el aumento de la expresión del gen $T L R 4$, el aumento de lípidos en sangre y de la producción de especies reactivas del oxígeno. Mientras que en los conejos AP/AAS tanto la expresión del gen TLR4 y la producción de ERO disminuyen, ello probablemente se debe al efecto antioxidante del AAS. Estos resultados son similares a los de Chen et al. (2013), que en conejos alimentados por dos meses con una dieta alta en colesterol $(5 \%)$ y grasa $(15 \%)$, reportaron una condición de hiperlipidemia y el aumento de la expresión de TLR4 en macrófagos de sangre. 
Obesidad, dislipidemia, inflamación, estrés oxidante, hígado graso no alcohólico (HGNA).

Por lo que respecta a la expresión del gen TLR4 en los conejos AAS, se observó que aumentó hasta 4 veces más que en los conejos AP/AAS, es probable que esta sobre-expresión sea debida al efecto prooxidante del AAS (Battaglia et al., 2005). Los resultados del aumento en la producción de ERO así como de la expresión del gen TLR4 en los conejos AAS, nos llevan a proponer que el uso de AAS continuo por tres meses, en conejos con dieta normal sin exceso de lípidos, no es aconsejable por su efecto pro-oxidante.

El aumento de la expresión del gen TLR4, tanto en presencia de ácidos grasos libres provenientes de la dieta rica en grasa, como en presencia de LPS ya ha sido reportado (Manček-Keberet al., 2015); en pacientes con artritis reumatoide se demostró cómo la proteína de membrana codificada por TLR4 puede reconocer lípidos oxidados de micro-vesículas externas y activar la vía de señalización de inflamación, como ocurre cuando reconoce LPS. Esta es una de las explicaciones de cómo se inicia la respuesta inflamatoria en presencia de ácidos grasos libres u oxidados. En ese sentido, los resultados de la expresión del gen TLR4 son similares a los que se encontraron en este trabajo, ya que dicha expresión así como la producción de ERO, aumentaron en los conejos jóvenes con dislipidemia.

\section{Agradecimientos:}

Agradecemos al Dr. Miguel Murguía Romero su colaboración en el análisis y discusión de resultados, la revisión y sus valiosos comentarios a este manuscrito, al Dr. Antonio Peña Díaz por todas las facilidades otorgadas para hacer este trabajo, y a dos revisores anónimos que hicieron observaciones a una versión previa del manuscrito que lo mejoraron substancialmente. Este trabajo fue financiado parcialmente por el proyecto PAPIIT IN226716 de la DGAPA-UNAM.

\section{Bibliografía}

Akarasereenont P, Bakhle YS, Thiemermann C y Vane JR. (1995). Cytokine-mediated induction of cyclooxygenase- 2 by activation of tyrosine kinase in bovine endothelial cells stimulated by bacterial lipopolysaccharide. British Journal of Pharmacology; 115: 401-408.
Alberti KG, Eckel RH, Grundy SM, Zimmet PZ, Cleeman J, Donato KA, Fruchart J, James W, Loria CM, Smith SC Jr. International Diabetes Federation Task Force on Epidemiology and Prevention, National Heart, Lung, and Blood Institute, American Heart Association, World Heart Federation, International Atherosclerosis Society, International Association for the Study of Obesity. (2009). Harmonizing the metabolic syndrome: a joint interim statement of the International Diabetes Federation Task Force on Epidemiology and Prevention; National Heart, Lung, and Blood Institute; American Heart Association; World Heart Federation; International Atherosclerosis Society; and International Association for the Study of Obesity. Circulation 120: 1640-1645.

Ayyadevara S, Bharill P, Dandapat A, Hu C, Khaidakov M, Mitra S, Shmooklwe Reis RJ y Mehta JL. (2013). Aspirin Inhibits Oxidant Stress, Reduces AgeAssociated Functional Declines, and Extends Lifespan of Caenorhabditis elegans. Antioxidants \& Redox Signaling; 18 (5):481-490.

Battaglia V, Salvi M y Toninello A. (2005). Oxidative stress is responsible for mitochondrial permeability transition induction by salicylate in liver mitochondria. Journal of Biological Chemistry; 280(40): 3386433872.

Calderón M, Peña A y Thomé PE. (2006). DhARO4, an amino acid biosynthetic gene, is stimulated by high salinity in Debaryomyces hansenii. Yeast; (23):725734 .

Cardoso AR, Kakimoto PA y Kowaltowski AJ. (2013). Diet-Sensitive Sources of Reactive Oxygen Species in Liver Mitochondria: Role of Very Long Chain AcylCoA Deshydrogenases. PLOS ONE; 8 (10): e77088.

Clària J, Lee MH y Serhan CN. (1996). Aspirin-Triggered Lipoxins (15-epi-LX) Are Generated by the Human Lung Adenocarcinoma Cell Line (A549)- Neutrophil Interactions and Are Potent Inhibitors of Cell Proliferation. Molecular Medicine; 2(5):583-596.

Cyrus T, Sung S, Zhao L, Funk CD, Tang S y Praticò D. (2002). Effect of Low-Dose Aspirin on Vascular Inflammation, Plaque Stability, and Atherogenesis in Low Density Lipoprotein Receptor-Deficient Mice. Circulation; 106:1282-1287.

Chen S, Lin G, Lei L, You X, Wu C, Xu W, Huang M, Luo L, Wang Z, Li Y, Zhao X, y Yan F. (2013). Hyperlipidemia Modifies Innate Immune Responses to Lipopolysaccharide via the TLR-NF- $\mathrm{KB}$ Signaling Pathway. Inflammation; 36(4): 968-976. 
Fang D, Yang S, Quan W, Jia H, Quan Z y Qu Z. (2014). Atorvastatin suppresses Toll-like receptor 4 expression y NF-kB activation in rabbit atherosclerotic plaques. European Review for Medical and Pharmacological Sciences; (18): 242-246.

Fields M, Lewis CG y Bureau I. (2001). Aspirin Reduces Blood Cholesterol in Copper-Deficient Rats: A Potential Antioxidant Agent? Metabolism; 50(5): 558561.

Furukawa S, Fujita T, Shimabukuro M, Iwaki M, Yamada Y, Nakajima Y, Nakayama O, Makishima M, Matsuda M, y Shimomura I. (2004). Increased oxidative stress in obesity and its impact on metabolic syndrome. $J$ Clin Invest; 114(12):1752-1761.

Gou J y Friedman SL. (2010). Toll-like receptor 4 signaling in liver injury and hepatic fibrogenesis. Fibrogenesis \& Tissue Repair; (3):21.

Hempel SL, Buettner GR, O`Malley YQ, Wessels DA, y Flaherty DM. (1999). DihydrofluoresceinDiacetate is superior for detecting intracellular oxidants: Comparision with 2'7' Dichlorodihydrofluorescein diacetate, 5 (and 6)-Carboxy-2'7' Dichlorodihydrofluorescein diacetate and Dihydrorhodamine 123. Free Radical Biology \& Medicine; 27(1-2): 146-159.

Hsieh PS, Jin JS, Chiang CF, Chan PC, Chen CH y Shih KC. (2009). COX-2-mediated Inflammation in Fat Is Crucial for Obesity-linked Insulin Resistance and Fatty Liver. Obesity; (17): 1150-1157.

Integrated DNA Technologies. (2017). www.idtdna.com/pages. Obtenido de https://www.idtdna.com/calc/analyzer

Kibbe, W. (2007). OligoCalc: an online oligonucleotide properties calculator. Obtenido de http://biotools.nubic.northwestern.edu/OligoCalc.html

Kourtis N y Tavernarakis N. (2011). Cellular stress response pathways and ageing: intricate molecular relationships. EMBO Journal; (30): 2520-2531.

Lee JY, Ye J, Gao Z, Youn H, Lee WH, Zhao L, Sizemore N y Hwang DH. (2003). Reciprocal Modulation of Toll-like Receptor-4 Signaling Pathways Involving MyD88 and Phosphatidylinositol 3-Kinase/AKT by Saturated and Polyunsaturated Fatty Acids. The Journal of Biological Chemistry; 278 (39): 37041- 37051.
Liu J, Zhuang ZJ, Bian D, Ma XJ, Xun YH, Yang WJ, Lou Y, Liu YL, Jia L, Wang Y, Zhu M, Ye DW, Zhou G, Lou GQ y Shi JP. (2014). Toll-like receptor-4 signalling in the progression of non-alcoholic fatty liver disease induced by high-fat and high-fructose diet in mice. Clinical and Experimental Pharmacology and Physiology; (41):482-488.

Mahmood KA, Ahmed JH y Jawad AM. (2009). Nonstereroidal anti-inflammatory drugs (NSAIDS), free radicals and reactive oxygen species (ROS): A review of literature. The Medical Journal of Basrah University; 27(1):46-53.

Manček-Keber M, Frank-Bertoncelj M, Hafner-Bratkovič I, Smole A, Zorko M, Pirher N, Hayer S, Kralj-Iglič V, Rozman B, Ilc N, Horvat S y Jerala R. (2015). Toll-like receptor 4 senses oxidative stress mediated by the oxidation of phospholipids in extracellular vesicles. Science Signaling; 8(381): 1-12.

McRae MP. (2008). Vitamin C supplementation lowers serum low-density lipoprotein cholesterol and triglycerides: a meta-analysis of 13 randomized controlled. Journal of Chiropractic Medicine; (7); $48-58$.

Milagro FI, Javier Campión J, y Martínez JA. (2006). Weight Gain Induced by High-Fat Feeding

Involves Increased Liver Oxidative Stress. Obesity; 14(7): 1118-1123.

Oliveira CP, da Costa Gayotto LC, Tatai C, Della Bina BI, Janiszewski M, Lima ES, Abdalla DS, Lopasso FP, Laurindo FR y Laudanna AA. (2002). Oxidative stress in the pathogenesis of nonalcoholic fatty liver disease, in rats fed with a choline-deficient diet. $J$ Cell Mol Med; 6 (3): 399-406.

Pagadala M, Zein CO y McCullough AJ. (2009) Predictors of Steatohepatitis and Advanced Fibrosis in Non-Alcoholic Fatty Liver Disease. Clin Liver Dis; (13): 591-606.

Paul-Clark MJ, Van Cao T, Moradi-Bidhendi N, Cooper D y Gilory DW. (2004). 15-epi-lipoxin A4-mediated Induction of Nitric Oxide Explains How Aspirin Inhibits Acute Inflammation. J Expe Med; 200(1):6978.

Petrosillo G, Portincasa P, Grattagliano I, Casanova G, Matera M, Ruggiero FM, Ferri y Paradies G. (2007). Mitochondrial dysfunction in rat with nonalcoholic fatty liver Involvemet of complex I, reactive oxygen species and cardiolipin. Biochimica et Biophysica Acta; (1726):1260-1267. 
Obesidad, dislipidemia, inflamación, estrés oxidante,

hígado graso no alcohólico (HGNA).

Podhaisky HP, Abate A, Polte T, Oberle OS y Schröder H. (1997). Aspirin protects endothelial cells from oxidative stress - possible synergism with vitamin E. FEBS Letters; (417): 349-351.

Ricciotti E y FitzGerald GA. (2011). Prostaglandins and Inflammation. Arterioscler Thromb Vasc Biol; 31(5): 986-1000.

Schmitt ME, Brown TA y Trumpower BL (1990) A rapid and simple method for preparation of RNA from Saccharomyces cerevisiae. Nucleic Acids Res; (18):3091-3092.

Song J, Ke SF, Zhou CC, Zhang SL, Guan YF, Xu TY, Sheng CQ, Wang P y Miao CY. (2013). Nicotinamide Phosphoribosyltransferase Is Required for the Calorie Restriction-Mediated Improvements in Oxidative Stress, Mitochondrial Biogenesis, and Metabolic Adaptation. J Gerontol A Biol Sci Med Sci; 69(1):4457.

Stojsavljevió S, Gomerčió Palčió M, Virovió Jukió L, Smirčió Duvnjak L y Duvnjak M. (2014). Adipokines and proinflammatory cytokines, the key mediators in the pathogenesis of nonalcoholic fatty liver disease. World Journal of Gastroenterology; 20(28):1807018091.

Suganami T, Tanimoto-Koyama K, Nishida J, Itoh M, Yuan X, Mizuari S, Kotani H, Yamaoka S, Miyake K, Aoe S, Kamei Y y Ogawa Y. (2007). Role of the Toll-like Receptor 4/NF-kappaB Pathway in Saturad Fatty AcidInduced Inflammatory Changes in the Interaction Between Adipocytes and Macrophages. Arterioscler Thromb Vasc Biol. 27(1):84-91. DOI: 10.1161/01

Tilg H. (2010). The Role of Cytokines in Non-Alcoholic Fatty Liver Disease. Digestive Diseases; 28(1): 179185.

Untergasser A, C. K.-S. (2012). Primer3-new capabilities and interfaeces. Obtenido de bioinfo.ut.ee: http://bioinfo.ut.ee/primer3-0.4.0/

Yu J, Ip E, De la Peña A, Hou JY, Sesha J, Pera N, Hall P, Kirsh R, Leclerq I y Farrell C. (2006). COX-2 Induction in Mice With Experimental Nutritional Steotohepatitis: Role as Pro-inflammatory Mediator. Hepatology; 43(4): 286-386. 